\title{
ON THE RANGES OF BIMODULE PROJECTIONS
}

\author{
ARISTIDES KATAVOLOS AND VERN I. PAULSEN
}

\begin{abstract}
We develop a symbol calculus for normal bimodule maps over a masa that is the natural analogue of the Schur product theory. Using this calculus we are able to easily give a complete description of the ranges of contractive normal bimodule idempotents that avoids the theory of $\mathrm{J}^{*}$-algebras. We prove that if $P$ is a normal bimodule idempotent and $\|P\|<2 / \sqrt{3}$ then $P$ is a contraction. We finish with some attempts at extending the symbol calculus to non-normal maps.
\end{abstract}

\section{INTRODUCTION}

Let $\mathcal{H}$ be a Hilbert space and let $B(\mathcal{H})$ denote the algebra of bounded linear operators on $\mathcal{H}$. If $P: B(\mathcal{H}) \rightarrow B(\mathcal{H})$ is a completely contractive idempotent, then it is known that its range $\mathcal{M}=P(B(\mathcal{H}))$ can be endowed with a triple product that makes it a ternary ring of operators (TRO). That is, there exists a unital $C^{*}$-algebra, $\mathcal{A}$, a self-adjoint idempotent $q \in \mathcal{A}$, and a complete onto isometry $\phi: \mathcal{M} \rightarrow q \mathcal{A}(1-q)$, such that the triple product on $\mathcal{M}$ is given by $\left\{m_{1}, m_{2}, m_{3}\right\}=\phi\left(m_{1}\right) \phi\left(m_{2}\right)^{*} \phi\left(m_{3}\right)$.

The presence of the map $\phi$ means that the triple product, in general, has little relation to the product inherited from $B(\mathcal{H})$. However, when $P$ is also assumed to be a bimodule map with respect to a masa in $B(\mathcal{H})$, then Solel So has shown that considerably more is true.

Let $\mathcal{D} \subseteq B(\mathcal{H})$ be a masa acting on a separable Hilbert space $\mathcal{H}$ and let $P: B(\mathcal{H}) \rightarrow B(\mathcal{H})$ be a contractive idempotent $\mathcal{D}$-bimodule map. In case $P$ is also $\mathrm{w}^{*}$-continuous, Solel $[\mathrm{So}$ ] proves that the range $\mathcal{M}=P(B(\mathcal{H}))$ is actually a ternary ring of operators (TRO) in the product inherited from $B(\mathcal{H})$, i.e., it satisfies $\mathcal{M} \mathcal{M}^{*} \mathcal{M} \subseteq \mathcal{M}$. We summarize this stronger condition by saying that $\mathcal{M}$ is a sub-TRO of $B(\mathcal{H})$.

We give a new proof of Solel's result, that does not need any $\mathrm{J}^{*}$-algebra theory, and in fact obtain the stronger conclusion that $\mathcal{M}$ must be the $\mathrm{w}^{*}$ closed sum of "full corners", $\mathcal{M}=\sum_{n} B\left(\mathcal{H}_{n}, \mathcal{K}_{n}\right)$ where $\left\{\mathcal{H}_{n}\right\}$ and $\left\{\mathcal{K}_{n}\right\}$ are pairwise orthogonal subspaces of $\mathcal{H}$ and $\mathcal{K}$ respectively. From this description of $\mathcal{M}$ it is immediate that $\mathcal{M} \mathcal{M}^{*} \mathcal{M} \subseteq \mathcal{M}$.

2000 Mathematics Subject Classification. Primary 46L15; Secondary 47L25.

Research supported in part by a grant from the University of Athens (A. Katavolos) and the NSF (V.I. Paulsen)

Preliminary versions of this work were announced by the authors in 2001 and 2002, in particular during the Workshop on Nonself-adjoint Operator Algebras held at the Fields Institute (see http://www.fields.utoronto.ca/audio/02-03/nsoa/paulsen/). 
In addition, representing $\mathcal{D}$ as the multiplication masa of a standard Borel space $(X, \mu)$, we show that the $\omega$-support (see [EKS]) of the range $\mathcal{M}$ of a $\mathrm{w}^{*}$-continuous idempotent $\mathcal{D}$-bimodule map (whether contractive or not) is $\omega$-open (as well as $\omega$-closed). It follows EKS that the reflexive cover $\operatorname{Ref}(\mathcal{M})$ of $\mathcal{M}$ is strongly reflexive.

We begin by examining the case when $\mathcal{H}=\ell^{2}$ and $\mathcal{D}=\ell^{\infty}$ in some detail. In this case every $\mathcal{D}$-bimodule map $\Phi$ is well-known to be given by Schur multiplication against a fixed matrix $A=\left(a_{i, j}\right)$, that is, $\Phi(X)=A * X=$ $\left(a_{i, j} x_{i, j}\right)$. We denote this map by $\Phi=S_{A}$.

Note that $\Phi \circ \Phi=\Phi$ is clearly equivalent to $a_{i, j}^{2}=a_{i, j}$ and hence each entry of $A$ must be either 0 or 1 . Thus every idempotent $\Phi$ can be identified in a one-to-one fashion with a subset $E \subseteq \mathbb{N} \times \mathbb{N}$ where $E=\left\{(i, j): a_{i, j}=1\right\}$ and we write $A=\chi_{E}$, where we regard the matrix $A$ as a function of two variables.

The problem of determining ranges of bounded bimodule projections, becomes one of determining which subsets of $\mathbb{N} \times \mathbb{N}$ give rise to bounded bimodule projections.

When a non-zero idempotent Schur product map has norm less than $2 / \sqrt{3}$, then Livshits Li] proves that it must actually be of norm 1 . We present a somewhat different proof of this result, based on a combinatorial "3 of 4" lemma, that gives a description of these sets that generalizes more readily from the case of discrete masa's to continuous masa's.

Very little is known about the structure of sets such that $\Phi$ is only a bounded projection, but we give, hopefully, a little insight into this problem.

In the third section, we use Haagerup's results Haa to develop a symbol calculus for $\mathrm{w}^{*}$-continuous bimodule maps over general masa's. One of the main advantages of our approach is that the symbol calculus allows proofs given in the discrete case to carry over to arbitrary masa's. In particular, we prove that every non-zero $\mathrm{w}^{*}$-continuous bimodule projection of norm less than $2 / \sqrt{3}$ is actually of norm 1 . Thus, the set of possible norms of $\mathrm{w}^{*}$-continuous bimodule projections is not a connected subset of the reals. It is not known if the same phenomenon holds for all bounded bimodule projections.

In the final section we study masa bimodule maps that are not $\mathrm{w}^{*}$ continuous. Solel [So] conjectures that the range of an idempotent contractive masa bimodule map $P$ will still be a sub-TRO even when $P$ is not $\mathrm{w}^{*}$-continuous. We use the actions of such maps on normalizers of the bimodule as an approach to the problem of extending our symbol calculus beyond the $\mathrm{w}^{*}$-continuous case.

If $P$ is a contractive idempotent (hence $\|P\|=1$ ) and its range is a $\mathcal{D}$-bimodule, then $P$ is automatically a $\mathcal{D}$-bimodule map ( $\underline{\mathrm{So}}]$ ).

The authors would like to thank Ken Davidson, Allan Donsig, Gilles Pisier and Ivan Todorov for various observations that have improved our exposition and results. 


\section{The Discrete Case}

In this section we develop the case where the masa is totally atomic, so our Hilbert space may be represented as $\ell^{2}$ and the masa as $\ell^{\infty}$ acting in the usual fashion as diagonal matrices. Identifying $\ell^{2}=L^{2}(\mathbb{N}, \mu)$, leads to the identification of $\ell^{\infty}=L^{\infty}(\mathbb{N}, \mu)$ acting as multiplication operators on this space of functions.

Definition 1. Let $X$ and $Y$ be sets and let $E \subseteq X \times Y$. We say that $E$ has the $\mathbf{3}$ of $\mathbf{4}$ property provided that given any distinct pair of points $x_{1} \neq x_{2}$ in $X$ and any pair of distinct points $y_{1} \neq y_{2}$ in $Y$, whenever 3 of the 4 ordered pairs $\left(x_{i}, y_{j}\right)$ belong to $E$ then the fourth ordered pair belongs to $E$ also.

Lemma 2. Let $X$ and $Y$ be sets and let $E \subseteq X \times Y$. If $E$ has the 3 of 4 property, then there exists an index set $T$, disjoint subsets $\left\{X_{t}\right\}_{t \in T}$ of $X$, and disjoint subsets $\left\{Y_{t}\right\}_{t \in T}$ of $Y$ such that

$$
E=\cup_{t \in T} X_{t} \times Y_{t} \text {. }
$$

Proof. Define a relation on $X$ by $x_{1} R x_{2}$ if and only if there exists $y \in Y$ such that $\left(x_{1}, y\right)$ and $\left(x_{2}, y\right)$ are both in $E$. The 3 of 4 property ensures that $R$ is transitive and hence is an equivalence relation on the subset $X_{0}=\{x \in$ $X:(x, y) \in E$ for some $y \in Y\}$ of $X$. Let $\left\{X_{t}\right\}_{t \in T}$ denote the collection of equivalence classes of $X_{0}$.

Define $Y_{t}=\left\{y \in Y:(x, y) \in E\right.$ for some $\left.x \in X_{t}\right\}$. Again the 3 of 4 property ensures that the sets $Y_{t}$ are disjoint with union equal to

$$
Y_{0}=\{y \in Y:(x, y) \in E \text { for some } x \in X\} .
$$

One final use of the 3 of 4 property shows that $E=\cup_{t \in T} X_{t} \times Y_{t}$.

The following result is also Lemma 3 of [Li], but the proof is short, so we include it.

Lemma 3. Let $A=\left(\begin{array}{ll}1 & 1 \\ 0 & 1\end{array}\right)$ and let $S_{A}: M_{2} \rightarrow M_{2}$ denote the map given as Schur product by $A$. Then $\left\|S_{A}\right\|=2 / \sqrt{3}$.

Proof. Since the unitaries are the extreme points of the unit ball of $M_{2}$, it is easily seen that $\left\|S_{A}\right\|=\sup _{\theta}\left\|\left(\begin{array}{cc}\cos \theta & \sin \theta \\ 0 & \cos \theta\end{array}\right)\right\|$ and the result follows by computing this supremum.

Theorem 4. Let $P: B\left(\ell^{2}\right) \rightarrow B\left(\ell^{2}\right)$ be a non-zero $\ell^{\infty}$-bimodule map that is idempotent, let $\mathcal{M}=P\left(B\left(\ell^{2}\right)\right)$ denote the range of $P$ and assume that $\|P\|<2 / \sqrt{3}$. Then:

(i) $P=S_{\chi_{E}}$ where $E=\cup I_{m} \times J_{m}$, with $\left\{I_{m}\right\}$ and $\left\{J_{m}\right\}$ countable collections of disjoint subsets of $\mathbb{N}$,

(ii) $\mathcal{M}=\sum_{m} \chi_{I_{m}} B\left(\ell^{2}\right) \chi_{J_{m}}$ and $\mathcal{M} \mathcal{M}^{*} \mathcal{M} \subseteq \mathcal{M}$,

(iii) $\|P\|=1$. 
Proof. The fact that $P=S_{\chi_{E}}$ for some set $E \subseteq \mathbb{N} \times \mathbb{N}$ was noted in the introduction. Choose any $x_{1} \neq x_{2}$ and $y_{1} \neq y_{2}$ in $\mathbb{N}$ and consider the compression of $P$ as a map from the span of $\left\{e_{x_{1}}, e_{x_{2}}\right\}$ to the span of $\left\{e_{y_{1}}, e_{y_{2}}\right\}$. Since $\|P\|<2 / \sqrt{3}$, by the above lemma $E$ will have the 3 of 4 property and hence by the first lemma be of the form given in (i). It is now obvious that $\mathcal{M}$ will have the form claimed in (ii). The second assertion in (ii) is immediate from this.

Alternatively, to see the second assertion in (ii), note that it is enough to assume that the matrix units $E_{i, j}, E_{k, l}$ and $E_{m, n}$ are in $\mathcal{M}$ and prove that $E_{i, j} E_{k, l}^{*} E_{m, n}$ is in $\mathcal{M}$. But this product will be 0 unless $j=l$ and $k=m$ in which case the product is $E_{i, n}$. However, in this case we have that $(i, j),(k, j),(k, n)$ belong to $E$ and so again by the 3 of 4 property $(i, n)$ is in $E$ and so $E_{i, n} \in \mathcal{M}$.

Finally, to prove (iii), let $\left\{e_{m}\right\}$ denote the usual basis of $\ell^{2}$, set $x_{i}=e_{m}$ when $i \in I_{m}$, set $y_{j}=e_{m}$ when $j \in J_{m}$ and note that $\chi_{E}(i, j)=\left\langle x_{i}, y_{j}\right\rangle$. Thus, $\|P\| \leq 1$ by the theorem characterizing the norms of Schur product maps, see for example $[\mathrm{Pa}$.

Theorem 4(iii), with a somewhat longer proof, is contained explicitly in Li] and (i) and (ii) can be obtained from the proof given there.

It is well known that the range of a completely contractive projection is completely isometrically isomorphic to a TRO on some Hilbert space $\mathcal{H}$. This result induces a triple product on the range, but it is generally not the triple product given by the original representation of the range as a subspace of $B(\mathcal{H})$. Note that by (ii), we have that the range of $P$ is a TRO in the original triple product, i.e., that the range is a sub-TRO of $B\left(\ell^{2}\right)$.

Remark 5. By the above result we see that the set of possible norms of bimodule projections does not contain the interval from 1 to $2 / \sqrt{3}$. This makes the structure of this set somewhat intriguing. Davidson has observed that the set of possible norms is closed under product and under the taking of suprema. By a result of Bhatia, Choi and Davis [BCD], the number 2 is one of the limit points of this set. Other than these facts, not much seems to be known about this set.

If we let $\Delta=\left\{E \subseteq \mathbb{N} \times \mathbb{N}: S_{\chi_{E}}\right.$ is bounded $\}$, then, by the results characterizing the norms of Schur product maps, it is easily seen that $E \in \Delta$ if and only if there exist bounded sequences of vectors, $\left\{x_{i}\right\}$ and $\left\{y_{j}\right\}$, such that $\chi_{E}(i, j)=\left\langle x_{i}, y_{j}\right\rangle$, but this characterization seems to be of little help in obtaining other conditions that characterize the sets in $\Delta$.

It is not hard to show that $\Delta$ is an algebra of sets and so contains the algebra generated by the sets given by the above theorem. It is not currently known whether $\Delta$ equals the latter algebra. 


\section{A Functional Calculus}

In the discrete case, every bounded bimodule map is given as a Schur product map and so is automatically $\mathrm{w}^{*}$-continuous, but this is not the case in general. In this section we develop a functional calculus in the nondiscrete case for $\mathrm{w}^{*}$-continuous bimodule maps that allows us to treat these exactly like Schur product maps and consequently obtain exact analogues of the results of the previous section.

This functional calculus is different from the one considered by Peller $[\mathrm{Pe}$, and appears to have recently been discovered independently by Shulman and Kissin $\underline{K S}$.

Let $\mathcal{D} \subseteq B(\mathcal{H})$ be a masa acting on a separable Hilbert space $\mathcal{H}$ and let $\Phi: B(\mathcal{H}) \rightarrow B(\mathcal{H})$ be a $\mathrm{w}^{*}$-continuous $\mathcal{D}$-bimodule map. Since $\Phi$ is a bounded $\mathcal{D}$-bimodule map, a result of R. Smith [Smi] (see also [DP]) shows that $\Phi$ must be completely bounded, and in fact $\|\Phi\|_{c b}=\|\Phi\|$.

Haagerup Haa has shown that a $\mathrm{w}^{*}$-continuous completely bounded $\mathcal{D}$ bimodule map such as $\Phi$ must be of the form

$$
\Phi(T)=\sum_{n=1}^{\infty} F_{n} T G_{n} \quad(T \in B(\mathcal{H}))
$$

for suitable $F_{n}, G_{n} \in \mathcal{D}$ satisfying $\left\|\sum F_{n} F_{n}^{*}\right\|<\infty$ and $\left\|\sum G_{n}^{*} G_{n}\right\|<\infty$.

Represent $\mathcal{D}$ as the multiplication masa of a standard (finite) Borel space $(X, \mu)$ acting on $\mathcal{H}=L^{2}(X, \mu)$. A standard null-set argument shows that we may choose two families $\left\{f_{n}\right\},\left\{g_{n}\right\}$ of Borel functions with $F_{n}=M_{f_{n}}$ and $G_{n}=M_{g_{n}}$ for each $n$, and such that the series $\sum\left|f_{n}(t)\right|^{2}$ and $\sum\left|g_{n}(t)\right|^{2}$ converge for all $t \in X$ boundedly and in $L^{2}$ norm.

It follows that the series

$$
\phi(s, t)=\sum_{n} f_{n}(s) g_{n}(t)
$$

converges pointwise everywhere to a Borel function.

Conversely, let $f=\left(f_{1}, f_{2}, \ldots\right)$ and $g=\left(g_{1}, g_{2}, \ldots\right)$ be (essentially) bounded weakly Borel measurable functions from $X$ into $\ell^{2}$. Since $\ell^{2}$ is separable, weak Borel measurability and strong Borel measurability are equivalent. Thus (i) each $f_{n}$ is an essentially bounded complex-valued function and (ii)

$$
\sup _{s \in X}\|f(s)\|_{2}^{2}=\sup _{s \in X} \sum_{n}\left|f_{n}(s)\right|^{2} \equiv B_{f}<\infty
$$

and $B_{g} \equiv \sup _{s \in X}\|g(s)\|_{2}^{2}<\infty$. It follows that

$$
\|f\|^{2} \equiv \int\|f(s)\|_{2}^{2} d \mu(s)=\sum_{n} \int\left|f_{n}(s)\right|^{2} d \mu(s)<\infty
$$

and similarly for $g$; hence the function

$$
\phi(s, t)=\langle f(s), \bar{g}(t)\rangle=\sum_{n} f_{n}(s) g_{n}(t)
$$


defines an element of the projective tensor product $L^{2}(X, \mu) \widehat{\otimes} L^{2}(X, \mu)$. Note that the series converges pointwise absolutely and boundedly and also in the projective norm. Thus the function $\phi$ is Borel on $X \times X$ and (essentially) bounded.

Denoting by $F_{n}$ (resp. $G_{n}$ ) the multiplication operator $M_{f_{n}}$ (resp. $M_{g_{n}}$ ) acting on $\mathcal{H}=L^{2}(X, \mu)$ we observe that for every $T \in B(\mathcal{H})$ the series $\sum_{n} F_{n} T G_{n}$ converges in the $\mathrm{w}^{*}$-topology. This is well known, and we sketch the proof for completeness.

The fact that the bounds $B_{f}$ and $B_{g}$ defined above are finite shows that the infinite matrices $\left[G_{1}, G_{2}, \ldots\right]^{t}$ and $\left[F_{1}, F_{2}, \ldots\right]$ define bounded operators $\mathcal{H} \rightarrow(\mathcal{H})^{(\infty)}$ and $(\mathcal{H})^{(\infty)} \rightarrow \mathcal{H}$ with norms $\sqrt{B_{g}}$ and $\sqrt{B_{f}}$ respectively. Thus the matrix product $\left[F_{1}, F_{2}, \ldots\right] T^{(\infty)}\left[G_{1}, G_{2}, \ldots\right]^{t}$, which we denote by $\Phi_{\phi}(T)$, defines an operator on $\mathcal{H}$ whose norm is at most $\|T\| \sqrt{B_{g} B_{f}}$. This operator is of course $\sum_{n} F_{n} T G_{n}$, the strong limit of its partial sums $\Phi_{N}(T)=\sum_{n=1}^{N} F_{n} T G_{n}$; but since these partial sums are uniformly bounded by $\|T\| \sqrt{B_{g} B_{f}}$, the convergence is actually ultrastrong.

Clearly $\Phi_{\phi}$ is a $\mathcal{D}$-bimodule map. We claim that it is $\mathrm{w}^{*}$-continuous. For this, it suffices to show that it is weak operator continuous on the unit ball $B(\mathcal{H})_{1}$ of $B(\mathcal{H})$. But if $T$ is a contraction, then for all $\xi, \eta \in \mathcal{H}$, we have

$$
\begin{aligned}
& \left|\left\langle\Phi_{\phi}(T) \xi, \eta\right\rangle-\left\langle\Phi_{N}(T) \xi, \eta\right\rangle\right|^{2}=\left|\sum_{n=N+1}^{\infty}\left\langle T G_{n} \xi, F_{n}^{*} \eta\right\rangle\right|^{2} \\
& \leq\left(\sum_{n=N+1}^{\infty}\left\|T G_{n} \xi\right\|^{2}\right)\left(\sum_{n=N+1}^{\infty}\left\|F_{n}^{*} \eta\right\|^{2}\right) \leq\|T\|^{2}\left(\sum_{n=N+1}^{\infty}\left\|G_{n} \xi\right\|^{2}\right) B_{f}\|\eta\|^{2} \\
& \leq\left(\sum_{n=N+1}^{\infty} \int\left|g_{n}(s)\right|^{2}|\xi(s)|^{2} d \mu(s)\right) B_{f}\|\eta\|^{2} .
\end{aligned}
$$

By dominated convergence, this tends to 0 as $N \rightarrow \infty$. It follows that the function $T \rightarrow\left\langle\Phi_{\phi}(T) \xi, \eta\right\rangle$ is the uniform limit on $B(\mathcal{H})_{1}$ of the weak operator continuous functions $T \rightarrow\left\langle\Phi_{N}(T) \xi, \eta\right\rangle$ and so is itself weak operator continuous.

Note that the map $\Phi_{\phi}$ acts as a multiplication operator on kernels of Hilbert Schmidt operators:

Proposition 6. Let $\phi(s, t)=\langle f(s), \bar{g}(t)\rangle$ where $f$ and $g$ are (essentially) bounded (weakly) Borel measurable functions from $X$ into $\ell^{2}$, and let $\Phi_{\phi}$ : $B(\mathcal{H}) \rightarrow B(\mathcal{H})$ be as above. The map $\Phi_{\phi}$ leaves the space of Hilbert Schmidt operators invariant. If $T \in B(\mathcal{H})$ is a Hilbert Schmidt operator with kernel $k \in L^{2}(X \times X)$, then $\Phi_{\phi}(T)$ has kernel $M_{\phi}(k)=\phi k$. Thus $\Phi_{\phi}$ acts on $L^{2}(X \times X)$ as multiplication by $\phi$.

Proof. Let $T=T_{k}$ be Hilbert Schmidt operator with kernel $k$. For $\xi, \eta \in \mathcal{H}$ we have 


$$
\begin{aligned}
\left\langle\Phi_{\phi}\left(T_{k}\right) \xi, \eta\right\rangle & =\left\langle\sum_{n=1}^{\infty} F_{n} T_{k} G_{n} \xi, \eta\right\rangle=\sum_{n=1}^{\infty}\left\langle T_{k} G_{n} \xi, F_{n}^{*} \eta\right\rangle \\
& =\sum_{n=1}^{\infty} \iint k(x, y) g_{n}(y) \xi(y) f_{n}(x) \overline{\eta(x)} d \mu(y) d \mu(x) \\
& =\iint \sum_{n=1}^{\infty} f_{n}(x) g_{n}(y) k(x, y) \xi(y) \overline{\eta(x)} d \mu(y) d \mu(x) \\
& =\iint \phi(x, y) k(x, y) \xi(y) \overline{\eta(x)} d \mu(y) d \mu(x) \\
& =\left\langle T_{\phi k} \xi, \eta\right\rangle
\end{aligned}
$$

and so $\Phi_{\phi}\left(T_{k}\right)=T_{\phi k}$.

The next result shows that a $\mathrm{w}^{*}$-continuous bimodule map $\Phi=\Phi_{\phi}$ determines the function $\phi$ essentially uniquely. Recall that a set $R \subseteq X \times X$ is called marginally null provided that there is a null set $N$ such that $R \subseteq(N \times X) \cup(X \times N)$. We say that two functions $\phi$ and $\psi$ on $X \times X$ are equal marginally almost everywhere and write $\phi=\psi$ m.a.e. provided that the set of points where they are not equal is marginally null.

Theorem 7. Let $\phi(s, t)=\langle f(s), \bar{g}(t)\rangle$ where $f$ and $g$ are (essentially) bounded (weakly) Borel measurable functions from $X$ into $\ell^{2}$, and let $\Phi_{\phi}$ : $B(\mathcal{H}) \rightarrow B(\mathcal{H})$ be as above. The following are equivalent:

(1) $\phi=0$ m.a.e.

(2) $\phi=0$ a.e.

(3) $\Phi_{\phi}=0$.

Proof. If the set

$$
R=\{(s, t) \in X \times X: \phi(s, t) \neq 0\}
$$

is contained in a set of the form $N \times X \cup X \times N$, where $N \subseteq X$ is null, then of course the product measure of $R$ is 0 . Thus (1) implies (2).

To show that (2) implies (3), observe that if $T=T_{k}$ is a Hilbert-Schmidt operator with (square-integrable) kernel $k$, then by Proposition $6 \Phi_{\phi}\left(T_{k}\right)=$ $T_{\phi k}$.

It follows that if $\phi=0$ a.e. then $\Phi_{\phi}\left(T_{k}\right)=0$ for any Hilbert-Schmidt operator $T_{k}$. Since $\Phi_{\phi}$ is $\mathrm{w}^{*}$-continuous, we obtain $\Phi_{\phi}=0$. Conversely if $\Phi_{\phi}=0$ then $\phi=0$ a.e.

It remains to prove that if the set $R$ is null, then it must be marginally null. For this, first observe that $R$ is (marginally equivalent to) a countable union of Borel rectangles. We use an argument of Arveson Arv: The set

$$
\left\{(\xi, \eta) \in \ell^{2} \times \ell^{2}:\langle\xi, \eta\rangle \neq 0\right\}
$$

is open in $\ell^{2} \times \ell^{2}$, and hence is a countable union $\cup_{n} U_{n} \times V_{n}$ of open rectangles. Letting $A_{n}=\left\{s \in X: f(s) \in U_{n}\right\}$ and $B_{n}=\left\{t \in X: g(t) \in V_{n}\right\}$ 
we see that, since $f, g: X \rightarrow \ell^{2}$ are Borel functions, the sets $A_{n}$ and $B_{n}$ are Borel and

$R=\{(s, t):\langle f(s), g(t)\rangle \neq 0\}=\bigcup_{n}\left\{(s, t):\langle f(s), g(t)\rangle \in U_{n} \times V_{n}\right\}=\bigcup_{n} A_{n} \times B_{n}$

as claimed. Thus if the product measure of $R$ is 0 we must have $\mu\left(A_{n}\right) \mu\left(B_{n}\right)=$ 0 for all $n \in \mathbb{N}$. If $N_{1}=\cup\left\{A_{n}: \mu\left(B_{n}\right) \neq 0\right\}$ and $N_{2}=\cup\left\{B_{n}: \mu\left(A_{n}\right) \neq 0\right\}$ then $\mu\left(N_{1}\right)=\mu\left(N_{2}\right)=0$ and

$$
R \subseteq N_{1} \times X \cup X \times N_{2}
$$

which completes the proof.

Definition 8. We let $N C B_{\mathcal{D}}(B(\mathcal{H}))$ denote the algebra of $w^{*}$-continuous $\mathcal{D}$-bimodule maps from $B(\mathcal{H})$ into itself. Given a $w^{*}$-continuous $\mathcal{D}$-bimodule map $\Phi$ as above we call the m.a.e. equivalence class of the function $\phi$ obtained above the symbol of $\Phi$ and denote it by $\Gamma(\Phi)$.

Corollary 9. Let $\mathcal{D}$ be represented as the multiplication masa on a standard Borel space $(X, \mu)$, let $\mathcal{B}_{\text {mae }}(X \times X)$ denote the algebra of bounded Borel functions on $X \times X$ modulo the marginally null functions. Then the map $\Gamma: N C B_{\mathcal{D}}(B(\mathcal{H})) \rightarrow \mathcal{B}_{\text {mae }}(X \times X)$ is a one-to-one homomorphism onto the subalgebra of functions that can be represented in the form $\phi(s, t)=$ $\langle f(s), g(t)\rangle$ for any bounded Borel measurable functions $f, g$ from $X$ into a separable Hilbert space.

We call the map $\Gamma$ the functional calculus for $\mathrm{w}^{*}$-continuous $\mathcal{D}$-bimodule maps.

Armed with the functional calculus, we can readily generalize the theorem of the previous section.

Theorem 10. Let $P: B(\mathcal{H}) \rightarrow B(\mathcal{H})$ be a $\mathcal{D}$-bimodule map that is idempotent and $w^{*}$-continuous, let $\mathcal{M}=P(B(\mathcal{H}))$ denote the range of $P$ and assume that $\|P\|<2 / \sqrt{3}$. Then:

(1) $\Gamma(P)=\chi_{E}$ where $E=\cup I_{m} \times J_{m}$, with $\left\{I_{m}\right\}$ and $\left\{J_{m}\right\}$ countable collections of disjoint Borel subsets of $X$,

(2) $\mathcal{M}=\sum_{m} \chi_{I_{m}} B\left(L^{2}\right) \chi_{J_{m}}$ and $\mathcal{M} \mathcal{M}^{*} \mathcal{M} \subseteq \mathcal{M}$,

(3) $\|P\|=1$.

Proof. Let $\Gamma(P)=\phi$, since $P \circ P=P$, by the functional calculus, $\phi^{2}=\phi$ marginally almost everywhere. Thus, we can pick a Borel subset $X_{1}$ of $X$ with $\mu\left(X \cap X_{1}^{c}\right)=0$ such that $\phi^{2}=\phi$ on $X_{1} \times X_{1}$. Hence, there is a Borel subset $E$ of $X_{1} \times X_{1}$ such that $\phi=\chi_{E}$ as functions on $X_{1} \times X_{1}$.

Thus, we may write $\chi_{E}(s, t)=\langle f(s), g(t)\rangle$ where $f, g$ are functions into a separable Hilbert space with $\|f(s)\|\|g(t)\|<2 / \sqrt{3}$ for all $s, t$.

By Lemma 3, if for any $s_{1} \neq s_{2}$ and $t_{1} \neq t_{2}$, we have that 3 of the 4 values $\left\langle f\left(s_{i}\right), g\left(t_{j}\right)\right\rangle$ are 1 , then the fourth value must also be 1 .

Hence the set $E$ satisfies the 3 of 4 property and so it must be a union of disjoint rectangles as in Lemma 2] Say, $E=\cup_{t \in T} I_{t} \times J_{t}$. 
It remains to be shown that the indexing set $T$ for the union is only countable and that each of the sets $I_{t}$ and $J_{t}$ are Borel. As in the proof of Theorem [7, the set $E=\left\{(s, t) \in X_{1} \times X_{1}: \phi(s, t) \neq 0\right\}$ can be written as a countable union of Borel rectangles, say $E=\cup_{n} A_{n} \times B_{n}$. Again, by the equivalence relation used to define the sets $I_{t}$ and $J_{t}$, if any point in a rectangle $A_{n} \times B_{n}$ is contained in $I_{t} \times J_{t}$ then $A_{n} \times B_{n} \subseteq I_{t} \times J_{t}$. Hence, each set $I_{t} \times J_{t}$ is the union of the at most countably many Borel rectangles that are contained in it and consequently is itself a Borel rectangle. Moreover, the set $T$ can be placed in a one-to-one correspondence with a partition of the integers, and hence is countable.

The remainder of the proof proceeds as in the proof of Theorem 4

As in the discrete case we have that $\mathcal{M}$ is a sub-TRO of $B\left(L^{2}\right)$, a result obtained by Solel [So].

Just as in the discrete case, very little is known about bimodule projections of greater norm.

More importantly, very little is known about contractive bimodule projections that are not $\mathrm{w}^{*}$-continuous. Such projections do exist, for example projections onto the masa $\mathcal{D}$ exist and when $\mathcal{D}$ is not discrete, these cannot be not $\mathrm{w}^{*}$-continuous. Solel $[\mathrm{So}$ ] conjectures that the range $\mathcal{M}$ of any contractive $\mathcal{D}$-bimodule projection satisfies $\mathcal{M M}^{*} \mathcal{M} \subseteq \mathcal{M}$.

We now turn our attention to further properties of the symbol calculus and of bounded $\mathrm{w}^{*}$-continuous idempotents.

Recall EKS that a subset $E \subseteq X \times X$ is said to be $\omega$-open if it differs from a countable union of Borel rectangles by a marginally null set, and is $\omega$-closed if its complement is $\omega$-open. Thus the set $E$ in Theorem 10 is $\omega$-open. The following Proposition strengthens this result and provides an alternative approach:

Proposition 11. Let $P \in N C B_{\mathcal{D}}(B(\mathcal{H}))$ be an idempotent with symbol $\Gamma(P)=\chi$. Then there exists an $\omega$-open and $\omega$-closed set $A \subseteq X \times X$ such that $\chi=\chi_{A}$ marginally almost everywhere.

Proof. Notice first that, in the terminology of [EKS], any element $\phi$ of the projective tensor product $L^{2}(X) \widehat{\otimes} L^{2}(X)$ is $\omega$-continuous, that is, $\phi^{-1}(U)$ is $\omega$-open in $X \times X$ for any open set $U \subseteq \mathbb{C}$ [EKS, Theorem 6.5].

Since $P$ is idempotent, so is its induced operator $M_{\chi}$ on $L^{2}(X \times X)$ (see Proposition [6). It follows that $\chi^{2}=\chi$ almost everywhere, i.e. the set

$$
B=\left\{(s, t): \chi^{2}(s, t)-\chi(s, t) \neq 0\right\}
$$

has product measure zero. On the other hand, since $\chi \in L^{2}(X) \widehat{\otimes} L^{2}(X)$, the function $\chi$ is $\omega$-continuous hence so is $\chi^{2}-\chi$. Thus $B$ must be $\omega$-open, in other words marginally equivalent to a countable union of rectangles. The fact that $B$ has product measure zero now implies, as noted earlier, that it is actually marginally null. Replacing $X$ by a suitable Borel subset $X_{1}$ such that $\mu\left(X \cap X_{1}^{c}\right)=0$, we may assume that $B=\emptyset$, i.e. that $\chi^{2}(s, t)=\chi(s, t)$ 
for all $(s, t) \in X \times X$. Thus letting $A=\chi^{-1}(\{1\})$ we see that $A$ is $\omega$-closed (since $\chi$ is $\omega$-continuous); but $A^{c}=\chi^{-1}(\{0\})$ is also $\omega$-closed.

It is shown in EKS that, given any space $\mathcal{S}$ of operators on $L^{2}(X)$, there exists an $\omega$-closed set $\Omega$, minimal up to marginally null sets, that supports all elements of $\mathcal{S}$, in the sense that if a Borel rectangle $\alpha \times \beta$ doesn't meet $\Omega$ then $M_{\beta} \mathcal{S} M_{\alpha}=\{0\}$ (here $M_{\beta} \in \mathcal{B}\left(L^{2}(X)\right.$ ) denotes the projection onto $\left.L^{2}(\beta)\right)$. This set is called the $\omega$-support of $\mathcal{S}$.

Proposition 12. Let $P \in N C B_{\mathcal{D}}(B(\mathcal{H}))$ be an idempotent with symbol $\Gamma(P)=\chi_{A}$. Then the set $A$ is (marginally equivalent to) the $\omega$-support of $\mathcal{M}=P(B(\mathcal{H}))$.

Proof. It is to be shown that a Borel rectangle $\alpha \times \beta$ has marginally null intersection with $A$ if and only if $M_{\beta} \mathcal{M} M_{\alpha}=\{0\}$. Note that the relation $M_{\beta} \mathcal{M} M_{\alpha}=\{0\}$ is equivalent to $M_{\beta} P(T) M_{\alpha}=0$ for all $T \in B(\mathcal{H})$. But, since the map $T \rightarrow M_{\beta} P(T) M_{\alpha}$ is $\mathrm{w}^{*}$-continuous, this is equivalent to $M_{\beta} P(T) M_{\alpha}=0$ for all Hilbert Schmidt $T=T_{k}$. By Proposition [6 $M_{\beta} P\left(T_{k}\right) M_{\alpha}=M_{\beta} T_{\chi k} M_{\alpha}=T_{h}$, where $h=\chi_{\alpha \times \beta} \chi_{A} k$. Thus the relation $M_{\beta} P(T) M_{\alpha}=0$ holds for all Hilbert Schmidt $T=T_{k}$ if and only if the set $(\alpha \times \beta) \cap A$ has product measure zero. But since this set is $\omega$-open, as shown in the proof of the last proposition this can only happen when $(\alpha \times \beta) \cap A$ is marginally null.

Since $A$ is $\omega$-open, it follows [EKS, Theorem 6.11] that the reflexive cover $\operatorname{Ref}(\mathcal{M})$ is in fact strongly reflexive, and is the strong closure of the linear span of the finite rank operators supported in $A$. In case $P$ is actually contractive, this of course follows immediately from the fact that the range of $P$ is a direct sum of full corners (Theorem 10).

\section{Module maps AND Normalizers}

We conjecture that it is possible to extend the functional calculus of the previous section beyond the $\mathrm{w}^{*}$-continuous module maps to a homomorphism from all of $C B_{\mathcal{D}}(B(\mathcal{H}))$ into an algebra of functions. When $\mathcal{D}$ is a continuous masa, then [HW] proves that not only is $C B_{\mathcal{D}}(B(\mathcal{H}))$ not abelian, but that its center is exactly the $\mathrm{w}^{*}$-continuous maps, $N C B_{\mathcal{D}}(B(\mathcal{H}))$.

Since the range of the homomorphism that we hope to construct would be contained in an abelian algebra, then necessarily this extended functional calculus would need to annihilate the commutator ideal. For this reason, we seek a representation of this algebra that annihilates the commutator ideal and a general understanding of the commutator ideal. We believe that the key to finding such a representation is to understand the action of module maps on normalizers.

Recall that an operator $T \in B(\mathcal{H})$ is said to normalize a masa $\mathcal{D} \subseteq B(\mathcal{H})$ if $T^{*} \mathcal{D} T \subseteq \mathcal{D}$ and $T \mathcal{D} T^{*} \subseteq \mathcal{D}$. If $T=V|T|$ is the polar decomposition, then $T$ normalizes $\mathcal{D}$ if and only if $|T| \in \mathcal{D}$ and $V$ normalizes $\mathcal{D}$. 
The set of all normalizers of $\mathcal{D}$ is a selfadjoint semigroup (under composition) and it contains $\mathcal{D}$. Therefore its norm-closed linear span is a unital $\mathrm{C}^{*}$-algebra, $\mathcal{B}$ say. If we decompose $\mathcal{D}$ into its totally atomic and continuous parts and let $\mathcal{H}=\mathcal{H}_{a} \oplus \mathcal{H}_{c}$ be the corresponding orthogonal decomposition of the underlying Hilbert space, then any partial isometry that normalizes $\mathcal{D}$ must leave each of these subspaces invariant. It follows that $\mathcal{B}$ has at most one nontrivial invariant projection, corresponding to this decomposition of $\mathcal{D}$. For a $\mathcal{B}$-projection must on the one hand commute with $\mathcal{D}$ hence must belong to it; on the other hand if a projection in $\mathcal{D}$ is invariant under all normalizers, then it must be trivial or the projection onto one of these parts. Thus, if we assume that $\mathcal{D}$ is either atomic or continuous, then the $\mathrm{w}^{*}$-closure of $\mathcal{B}$ is $B(\mathcal{H})$. We will show below that $\mathcal{B}$ is always proper.

When $\mathcal{D}$ is atomic, then it is clear that $\mathcal{B}$ contains all the corresponding matrix units and hence all of the compact operators.

Remark 13. If $\mathcal{D}$ is continuous, does $\mathcal{B}$ contain compact operators? Since $\mathcal{B}$ is irreducible, it follows that if it contains some nonzero compact operator then it must contain them all; for the set of all compact operators in $\mathcal{B}$ is then a nonzero ideal of the irreducible algebra $\mathcal{B}$, hence must itself be irreducible.

Proposition 14. Let $\Phi \in C B_{\mathcal{D}}=C B_{\mathcal{D}}(B(\mathcal{H}))$. For each normalizer $T$ of $\mathcal{D}$ there exists $D_{T} \in \mathcal{D}$ such that

$$
\Phi(T)=D_{T} T .
$$

In fact $D_{T}$ depends only on the partially isometric factor $V$ in the polar decomposition $T=V|T|$ of $T$.

Thus $\Phi(V f(|T|))=D_{T} V f(|T|)$ for any Borel function defined on $\sigma(|T|)$.

Proof. Since $V$ itself normalizes the masa while $|T|$ belongs to it, $\Phi(V|T|)=$ $\Phi(V)|T|$ by the module property of $\Phi$. Hence it suffices to deal with a normalizing partial isometry $V$. Now for each $D \in \mathcal{D}$ the element

$$
\Delta(V)=V^{*} D V
$$

belongs to $\mathcal{D}$, and so

$$
\begin{aligned}
V^{*} D & =V^{*}\left(V V^{*}\right) D=V^{*} D V V^{*}=\Delta(V) V^{*} \\
\text { and } \quad V \Delta(V) & =\left(V V^{*}\right) D V=D V V^{*} V=D V
\end{aligned}
$$

Therefore

$$
\begin{aligned}
\Phi(V) V^{*} D & =\Phi(V) \Delta(V) V^{*}=\Phi(V \Delta(V)) V^{*} \\
& =\Phi(D V) V^{*}=D \Phi(V) V^{*} .
\end{aligned}
$$

We have shown that $\Phi(V) V^{*}$ commutes with $\mathcal{D}$ and hence there exists $D_{V} \in$ $\mathcal{D}$ so that $\Phi(V) V^{*}=D_{V}$ hence

$$
D_{V} V=\Phi(V) V^{*} V=\Phi\left(V V^{*} V\right)=\Phi(V)
$$

as required. 
The above result does provide some sort of "symbol" for $\Phi$. If we assume that we are in the situation of the last section, namely, that $\mathcal{H}=L^{2}(X, \mu)$, and that $\Phi$ is a $\mathrm{w}^{*}$-continuous $L^{\infty}$-module map with symbol $\phi(s, t)$, then given any function $h$ on $X$ that gives rise to a bounded composition operator $C_{h}$ on $\mathcal{H}$, it is easy to see that $\Phi\left(C_{h}\right)=M_{k} C_{h}$, where $k(s)=\phi(s, h(s))$ a.e. This follows from the easily verified fact that $M_{f} C_{h} M_{g}=M_{k} C_{h}$ where $k(s)=f(s) g(h(s))$ a.e. and that $\Phi$ can be represented as a sum of such elementary operators.

Thus, the function $k$ tells us the value of the symbol restricted to the graph of $h$.

By the above result, even in the case that $\Phi$ is not $\mathrm{w}^{*}$-continuous, we see that for each bounded composition operator it is still true that $\Phi\left(C_{h}\right)=$ $M_{k} C_{h}$ for some function $k$ and the problem becomes, whether or not we can construct a symbol $\phi(s, t)$ such that $k(s)=\phi(s, h(s))$ holds for every $h$ that gives rise to a bounded composition operator.

For the following results, the reader should keep in mind that it is quite likely that $\mathcal{B}=\mathcal{B}+\mathcal{K}$.

Proposition 15. Let $\mathcal{B} \subseteq B(\mathcal{H})$ be the $C^{*}$-algebra generated by the normalizers of $\mathcal{D}$ and let $\mathcal{K}$ denote the ideal of compact operators. Then completely bounded $\mathcal{D}$-bimodule maps leave $\mathcal{B}, \mathcal{K}$ and $\mathcal{B}+\mathcal{K}$ invariant and the algebra obtained by restricting the $\mathcal{D}$-bimodule maps to any of these subspaces is commutative.

Proof. The fact that $\mathcal{B}$ is left invariant by every $\mathcal{D}$-bimodule map follows from Proposition 14. Let $\Phi_{1}, \Phi_{2} \in C B_{\mathcal{D}}(B(\mathcal{H}))$. If $T \in B(\mathcal{H})$ is a normalizer of $\mathcal{D}$, by Proposition 14 there exist $D_{1}, D_{2} \in \mathcal{D}$ s.t.

$$
\Phi_{1}(T)=D_{1} T, \quad \Phi_{2}(T)=D_{2} T .
$$

Since $\mathcal{D}$ is abelian, it follows that

$$
\begin{aligned}
\Phi_{1}\left(\Phi_{2}(T)\right) & =\Phi_{1}\left(D_{2} T\right)=D_{2} \Phi_{1}(T)=D_{2} D_{1} T \\
& =D_{1} \Phi_{2}(T)=\Phi_{2}\left(D_{1} T\right)=\Phi_{2}\left(\Phi_{1}(T)\right) .
\end{aligned}
$$

Thus the commutator $\Phi_{1} \circ \Phi_{2}-\Phi_{2} \circ \Phi_{1}$ vanishes on normalizers; hence it vanishes on $\mathcal{B}$ by linearity and continuity.

Hence, the algebra obtained by restricting $\mathcal{D}$-bimodule maps to $\mathcal{B}$ is commutative.

Proposition [6] shows that every normal bimodule map leaves $\mathcal{K}$ invariant. If we decompose an arbitrary bimodule map into its normal and singular part, then the singular part annihilates $\mathcal{K}$ and so also leaves it invariant. Thus, $\mathcal{K}$ is left invariant by every $\mathcal{D}$-bimodule map and the algebra obtained by restricting $\mathcal{D}$-bimodule maps to $\mathcal{K}$ is exactly $N C B_{\mathcal{D}}(B(\mathcal{H}))$.

The result for $\mathcal{B}+\mathcal{K}$ follows from the first two parts.

Corollary 16. The restriction maps

$$
R_{1}: C B_{\mathcal{D}}(B(\mathcal{H})) \rightarrow C B_{\mathcal{D}}(\mathcal{B}):\left.\Phi \rightarrow \Phi\right|_{\mathcal{B}}
$$


and

$$
R_{2}: C B_{\mathcal{D}}(B(\mathcal{H})) \rightarrow C B_{\mathcal{D}}(\mathcal{B}+\mathcal{K}):\left.\Phi \rightarrow \Phi\right|_{\mathcal{B}+\mathcal{K}}
$$

are onto, quotient maps that vanish on the commutator ideal of $C B_{\mathcal{D}}(B(\mathcal{H}))$.

Proof. The fact that $R_{1}$ vanishes on commutators is clear. By Wittstock's extension theorem for completely bounded bimodule maps [W], every map in $C B_{\mathcal{D}}(\mathcal{B})$ has an extension to a map of the same completely bounded norm in $C B_{\mathcal{D}}(B(\mathcal{H}))$. The fact that an extension exists means that $R_{1}$ is onto, and the fact that the $(\mathrm{cb})$ norm remains the same means that $R_{1}$ is a (complete) quotient map.

The proof for $\mathcal{B}+\mathcal{K}$ is identical.

Conjecture. We conjecture that the kernels of $R_{1}$ and of $R_{2}$ are both equal to the (closed) commutator ideal.

The above result also provides some insight into the structure of $\mathcal{B}$.

Corollary 17. When $\mathcal{D}$ is not totally atomic, the $C^{*}$-algebras $\mathcal{B}$ and $\mathcal{B}+\mathcal{K}$ are proper $\left(w^{*}\right.$-dense) subalgebras of $B(\mathcal{H})$.

Proof. If $\mathcal{B}+\mathcal{K}$ were equal to $B(\mathcal{H})$ then all $\mathcal{D}$-bimodule maps would commute. But this is not the case when $\mathcal{D}$ is not totally atomic by $\mathrm{HW}$. Hence $\mathcal{B}$ is also proper in this case.

The fact that the set of $\mathcal{D}$-bimodule maps is non-commutative when $\mathcal{D}$ is not totally atomic can also be seen directly. For example, if $\Phi_{1}, \Phi_{2}$ are two (norm-continuous) distinct projections onto the masa itself, then their commutator is their difference. To see that two such distinct projections exist it suffices, by splitting $\mathcal{D}$ into its atomic and continuous part, to assume $\mathcal{D} \simeq L^{\infty}[0,1]$ (see for example Tak, Theorem III.1.22). For this case two distinct projections onto $\mathcal{D}$ can be found for example in Dav, Algebras, 8.9 and 8.10 .

The above proof does not apply to the totally atomic case, since in this case the $\mathcal{D}$-bimodule maps do commute. Moreover, as remarked above, $\mathcal{B}$ contains all compacts. However, another argument shows that $\mathcal{B}$ is still a proper subalgebra of $B(\mathcal{H})$. We argue for the case that $\mathcal{D}=\ell^{\infty}$.

In this case, we have that $\mathcal{B} \subseteq B\left(\ell^{2}\right)$ is the $\mathrm{C}^{*}$-algebra generated by the diagonal $\mathcal{D}=\ell^{\infty}$ and its normalizers. It is convenient to label the basis of $\ell^{2}$ as $e_{0}, e_{1}, \ldots$. For each $A=\left[a_{i, j}\right] \in B\left(\ell^{2}\right)$, we denote by $a_{n}(n \in \mathbb{Z})$ its $n$-th diagonal, i.e. $a_{n}=\left(a_{i, j}\right)_{i-j=n} \in \ell^{\infty}$, so that formally $A=\sum_{n \in \mathbb{Z}} M_{a_{n}} S_{n}$ where $M_{a} \in B\left(\ell^{2}\right)$ is the operator of multiplication by $a \in \ell^{\infty}$ and we set $S_{n}=S^{n}$ for $n \geq 0$ and $S_{n}=\left(S^{*}\right)^{-n}$ for $n \leq 0$, where $S$ is the unilateral shift.

Lemma 18. Let $m: \ell^{\infty} \rightarrow \mathbb{C}$ be a Banach limit, i.e. a translation invariant state. If $A=\sum_{|n| \leq N} M_{a_{n}} S_{n}$ has finitely many nonzero diagonals, we set $\Psi_{o}(A)=\sum_{|n|<N} m\left(a_{n}\right) S_{n}$. Then $\Psi_{o}$ extends to a completely positive projection $\Psi: B\left(\ell^{2}\right) \rightarrow B\left(\ell^{2}\right)$ onto the Toeplitz operators. 
Proof. For $A \in B(\mathcal{H})$, consider the sesquilinear form

$$
\mathcal{H} \times \mathcal{H} \rightarrow \mathbb{C}:(x, y) \rightarrow m\left(\left(\left\langle A S^{n} x, S^{n} y\right\rangle\right)_{n \in \mathbb{N}}\right) .
$$

Since

$$
\left|m\left(\left(\left\langle A S^{n} x, S^{n} y\right\rangle\right)_{n \in \mathbb{N}}\right)\right| \leq\|m\| \sup _{n \in \mathbb{N}}\left|\left\langle A S^{n} x, S^{n} y\right\rangle\right| \leq\|A\|\|x\|\|y\|,
$$

(because $\|m\|=1$ and $\left\|S^{n}\right\|=1$ ) it is clear that this form is bounded, so there exists a unique $\Psi(A) \in B(\mathcal{H})$ such that

$$
\langle\Psi(A) x, y\rangle=m\left(\left(\left\langle A S^{n} x, S^{n} y\right\rangle\right)_{n \in \mathbb{N}}\right) .
$$

Note that the $k$-th diagonal of $A$ is the sequence $a_{k}=\left(\left\langle A e_{k+j}, e_{j}\right\rangle\right)_{j \in \mathbb{N}}$. Now for all $i, k \in \mathbb{N}$, the sequence $\left(\left\langle A e_{k+i+n}, e_{i+n}\right\rangle\right)_{n \in \mathbb{N}}$ is a translate of the sequence $a_{k}$, and so they have the same mean. Thus

$$
\left\langle\Psi(A) e_{k+i}, e_{i}\right\rangle=m\left(\left(\left\langle A S^{n} e_{k+i}, S^{n} e_{i}\right\rangle\right)_{n}\right)=m\left(\left(\left\langle A e_{n+k+i}, e_{n+i}\right\rangle\right)_{n}\right)=m\left(a_{k}\right)
$$

hence the $k$-th diagonal of $\Psi(A)$ is the constant sequence $\left(m\left(a_{k}\right), m\left(a_{k}\right), \ldots\right)$. Therefore $\Psi$ extends $\Psi_{o}$.

It is clear that $\Psi$ is a linear projection onto the set of Toeplitz operators. Finally, $\Psi$ is $k$-positive for all $k \in \mathbb{N}$. Indeed if $\left[A_{i j}\right] \in M_{k}(B(\mathcal{H}))$ is positive and $x=\left(x_{i}\right) \in \mathcal{H}^{k}$, we have

$$
\begin{aligned}
& \left\langle\left[\Psi\left(A_{i j}\right)\right] x, x\right\rangle=\sum_{i j}\left\langle\Psi\left(A_{i j}\right) x_{j}, x_{i}\right\rangle=\sum_{i j} m\left(\left(\left\langle A_{i j} S^{n} x_{j}, S^{n} x_{i}\right\rangle\right)_{n}\right) \\
& =m\left(\left(\sum_{i j}\left\langle A_{i j} S^{n} x_{j}, S^{n} x_{i}\right\rangle\right)_{n}\right)=m\left(\left(\left\langle\left[A_{i j}\right] \tilde{S}^{n} x, \tilde{S}^{n} x\right\rangle\right)_{n}\right) \geq 0
\end{aligned}
$$

(where $\tilde{S}^{n} x=\left(S^{n} x_{1}, S^{n} x_{2}, \ldots, S^{n} x_{k}\right)$ ).

Proposition 19. Let $\mathcal{B}$ and $\Psi$ be as above. The image $\Psi(\mathcal{B})$ contains only Toeplitz operators with continuous symbol; hence $\mathcal{B}$ cannot be all of $B\left(\ell^{2}\right)$.

Proof. Given a partially isometric normalizer $V$ of $\mathcal{D}$ let $\left(v_{n}\right)$ be its sequence of diagonals. Note that since $V$ has at most one 1 per row and column the (coordinatewise) sum $\sum_{n \in \mathbb{Z}} v_{n}$ is dominated by the vector $(2,2, \ldots) \in \ell^{\infty}$. It follows that for each $N \in \mathbb{N}$,

$$
0 \leq \sum_{|n| \leq N} m\left(v_{n}\right)=m\left(\sum_{|n| \leq N} v_{n}\right) \leq m((2,2, \ldots))=2
$$

and so $0 \leq \sum_{n \in \mathbb{Z}} m\left(v_{n}\right) \leq 2$. It follows that $\Psi(V)=T_{f}$ where $\sum|\hat{f}(n)| \leq 2$ and so $f$ is continuous (its Fourier series converges absolutely).

Consequently for any diagonal operator $D$ of norm $M$ say, the sum of the absolute values of the diagonals of $D V$ will be dominated by the vector $(2 M, 2 M, \ldots)$ and so $\Psi(D V)=T_{f}$ where $\sum_{n}|\hat{f}(n)| \leq 2 M$. Hence $f$ will be 
a continuous function on the circle. By linearity and continuity of $\Psi$, the result follows.

\section{REFERENCES}

[Arv] W.B. Arveson, Operator algebras and invariant subspaces, Ann. Math., 100(2):433$532,1974$.

[BCD] R. Bhatia, M.D. Choi and C. Davis, Comparing a matrix to its off-diagonal part. The Gohberg anniversary collection, Vol. I (Calgary, AB, 1988), 151-164,Oper. Theory Adv. Appl., 40, Birkhauser, Basel, 1989.

[Dav] Kenneth R. Davidson. Nest algebras, volume 191 of Pitman Research Notes in Mathematics Series. Longman Scientific \& Technical, Harlow, 1988. Triangular forms for operator algebras on Hilbert space.

[DP] K.R. Davidson \& S.C. Power, Isometric automorphisms and homology for nonself adjoint operator algebras, Quarterly J. Math.42 (2):271-292, 1991.

[EKS] J.A. Erdos, A. Katavolos and V.S. Shulman, Rank one subspaces of Bimodules over Maximal Abelian Selfadjoint Algebras, J. Funct. Anal. 157 (2): 554-587, 1998.

[Haa] Uffe Haagerup, Decomposition of completely bounded maps on operator algebras, Unpublished manuscript.

[HW] H.Hofmeier and G. Wittstock, A bicommutant theorem for completely bounded module homomorphisms, Math. Ann. 308(1997), 141-154.

[KS] E. Kissin and V.S. Shulman, work in progress (private communication).

[Li] L. Livshits, A note on 0-1 Schur multipliers, Lin. Alg. Appl. 222(1995), 15-22.

$[\mathrm{Pa}]$ V.I. Paulsen, Completely bounded maps and operator algebras, Cambridge University Press, 2003.

[Pe] V.V. Peller, Hankel operators in the theory of perturbations of unitary and selfadjoint operators. (Russian) Funktional. Anal. i Prilozhen., 19 (2): 37-51, 1985. English translation: Functional Anal. Appl. 19 (2): 111-123, 1985.

[Smi] R. R. Smith, Completely bounded module maps and the Haagerup tensor product, J. Funct. Anal., 102(1):156-175, 1991.

[So] B. Solel, Contractive projections onto bimodules of von Neumann algebras, J. London Math. Soc. (2) 45: 169-179, 1992.

[Tak] Masamichi Takesaki. Theory of operator algebras. I. Springer-Verlag, New York, 1979.

[W] G. Wittstock, Extensions of completely bounded module morphisms, Proc. of a conference on operator algebras and group representations, Neptun, Pitman, 1983.

Department of Mathematics, University of Athens, Athens, GreeCE

E-mail address: akatavol@math.uoa.gr

Department of Mathematics, University of Houston, Houston, Texas 772043476, U.S.A.

E-mail address: vern@math.uh.edu 